\title{
Associations between cardiorespiratory fitness and muscle strength with body composition among adults
}

\author{
NADIA BABAEI ${ }^{1, A, ~ B}$, SAMIRA DAVARZANI ${ }^{1, D, F}$, MOJDEH EBADITABAR $^{1, c}$, \\ KUROSH DJAFARIAN ${ }^{2, B}$ E, SAKINEH SHAB-BIDAR ${ }^{1, B, D, E}$ \\ ORCID ID: 0000-0001-8630-6155
}

\author{
${ }^{1}$ Department of Community Nutrition, School of Nutritional Sciences and Dietetics, Tehran University \\ of Medical Sciences (TUMS), Tehran, Iran \\ ${ }^{2}$ Department of Clinical Nutrition, School of Nutritional Sciences and Dietetics, Tehran University \\ of Medical Sciences (TUMS), Tehran, Iran
}

A - Study Design, B - Data Collection, C - Statistical Analysis, D - Data Interpretation, E - Manuscript Preparation, F - Literature Search, G - Funds Collection

Summary Background. Cardiorespiratory fitness (CRF) was reported to be associated with anthropometric measurements, including body mass, body mass index (BMI), fat mass (FM) and fat free mass (FFM). This study aimed to investigate the impact of FM and FFM on muscle strength and CRF.

Material and methods. This study was carried out on 270 randomly selected adults in Tehran, Iran. Anthropometric measurements, including weight, BMI, FM and FFM, were assessed using a bio-impedance analyzer (BIA) (InBody 720, Biospace, Tokyo, Japan). Muscle strength and CRF were assessed by handgrip dynamometer and Bruce graded treadmill protocol, respectively. The International Physical Activity Questionnaire (IPAQ) was used to measure health-related physical activity. A p-value less than 0.05 was statistically significant. Results. Significant associations were found between maximum oxygen uptake ( $\mathrm{VO}_{2}$ max) and muscle strength with FM and FFM $(p<0.001)$. After adjusting for confounders, the association of $\mathrm{VO}_{2}$ max with FFM remained significant $(p<0.001)$. Muscle strength was significantly associated with FM $(p=0.036)$ and FFM $(p<0.001)$ after adjustment for confounders.

Conclusions. High FM was associated with poor CRF, and low fat-free mass was related to poor muscle strength. FM and FFM can be good indices for CRF fitness in adults. Further longitudinal studies are needed to confirm these findings.

Key words: cardiorespiratory fitness, muscle strength, body composition.

Babaei N, Davarzani S, Ebaditabar M, Djafarian K, Shab-Bidar S. Associations between cardiorespiratory fitness and muscle strength with body composition among adults. Fam Med Prim Care Rev 2021; 23(2): 144-150, doi: https://doi.org/10.5114/fmpcr.2021.105905.

\section{Background}

Physical fitness is a state of functional capacity and well-being, and it is influenced by nutrition, physical fitness, muscle strength and aerobic capacity $[1,2]$. Muscle strength and cardiorespiratory fitness (CRF) are the two best main measurements of evaluation of physical performance and fitness [3]. Lower levels of CRF and grip strength are reported to be directly related to an increase in all-cause mortality risk [4-7]. Aerobic capacity or CRF is related to the capacity of circulatory and respiratory systems, which supply oxygen to the muscles during moderate to intense exercises $[8,9] . \mathrm{VO}_{2}$ max, which is considered as a gold standard for measuring CRF, is the maximal rate of oxygen consumption that could be used in strenuous activities [10]. Moreover, the upper and lower limits of body strength significantly influence one's ability to perform physical activity $[11,12]$. Handgrip strength is used in observational studies and clinical trials as an indicator of muscle strength [13].

On the other hand, anthropometric measurements, including BMI, fat mass (FM) and fat free mass (FFM), are indicators of body fitness [14-16]. The association between body composition with physiological traits, such as CRF and muscle grip, has been reported in several studies [17-20]. Most evidence has demonstrated that muscle mass is decreased with aging and leads to a decrease in muscle strength [20-22]. An inverse association was also found between body fat mass with muscular fitness and CRF [23-26]. Most studies have reported on the as- sociation between some certain measurements of body composition, such as BMI and waist circumference (WC), with physical performance $[17,27,28]$. There are limited studies which have investigated the association between FFM, FM and physical performance tests [29-32]. Thus, this study aimed to investigate the association of FM and FFM with CRF and muscle strength in Iranian adults.

\section{Material and methods}

\section{Subjects}

This cross-sectional study was carried out from May to September 2018 on 270 randomly selected Iranian adults (115 men and 155 women) from 18 to 70 years of age. The subjects were recruited from volunteers informed by advertisements through social media of the Tehran University of Medical Sciences. The sample size was calculated using the correlation coefficient between CRF and BF ( $r=0.31)$, which was reported in a previous study [33]. Participants were excluded from the study if they: 1) were an alcoholic or drug addict; 2) had been diagnosed with certain diseases, including heart, kidney, liver and pulmonary diseases; 3) were pregnant or lactating women and had restricted conditions for graded exercise treadmill tests (including asthma, MI, inflammation, recent history of hospitalization, inability to walk, arthritis problems with $\operatorname{limb}$ and disability). All participants signed the consent form before participation in the 
study. We set a scheduled time to start some of the tests for each participant at the Tehran University of Medical Sciences (Public Health Laboratory). The study participants were residents in the same area in Tehran (Latitude: $35^{\circ} 44^{\prime} \mathrm{N}$, Longitude: $51^{\circ} 30^{\prime} \mathrm{E}$ ). Data collection included completion of physical measurements (physical fitness, physical activity and some anthropometric data).

\section{Physical fitness measurements}

\section{Cardiorespiratory fitness}

CRF was determined through $\mathrm{VO}_{2}$ max, obtained from a graded exercise test on a treadmill utilizing the Bruce protocol [34]. The participants performed the Bruce graded treadmill protocol to complete exhaustion while the speed and incline of the treadmill were being increased every three minutes. The treadmill was started at $2.74 \mathrm{~km} / \mathrm{hr}$. $(1.7 \mathrm{mph})$ and at an incline of $10 \%$. At three-minute intervals, the incline of the treadmill was increased by $2 \%$, and the speed was increased based on the Bruce protocol. The test stopped when the subject could not continue due to fatigue or pain or due to any other medical indications. Respiratory gases were analyzed during the exercise protocol by MetaLyzer3B. The analyzer recorded and displayed data every 10 seconds. The data collected was stored using the Cortex MetaLyzer3B program, which has been validated applying the Douglas bag technique as the criterion method [35]. CRF was determined as $\mathrm{L} / \mathrm{min}$ and $\mathrm{ml} / \mathrm{kg} / \mathrm{min}$ with this program.

\section{Muscle strength}

Upper muscle strength was measured using an adjustable digital hand dynamometer in a normal sitting position. Grip strength is a reliable indicator of physical performance, especially muscle strength [36]. Handgrip strength is a predictor of upper body strength. Participants rotated their shoulders in a natural position and flexed their elbow to $90^{\circ}$. After obtaining proper settings for the arm and wrist in accordance with the protocol, force was exerted on a targeting sequence as hard as they could, three times for the right and left hands. The average of the tests in both hands was considered for analysis.

\section{Physical activity}

The short version of the International Physical Activity Questionnaire (IPAQ) was used to measure health-related physical activity [37]. The IPAQ questionnaires have been validated in 12 countries [38].

\section{Anthropometric measurements and body compo- sition}

Height was measured with a calibrated tape line fastened to a wall and with the participant wearing no shoes by a stadiometer (Seca, Germany) with a precision of $0.5 \mathrm{~cm}$. Weight, BMI, waist-hip ratio and WC were measured using a validated scale and tape line. Body composition, including body fat and body muscle percent, were also measured using a bio impedance analyzer (BIA) (InBody 720, Biospace, Tokyo, Japan). For this analysis, all patients were asked to follow these conditions before measurement: no food intake for at least 4 hours, no physical activity for at least 8 hours, no coffee or alcoholic beverage consumption for at least 12 hours, as well as with a restriction of no diuretic drugs use for at least 24 hours [39].

\section{Statistical analyses}

Analyses were carried out using the Statistical Package of the Social Sciences (SPSS version 25; SPSS Inc). Descriptive statistics of anthropometric measurements, physical fitness tests and body composition of the study samples were calculated as means and SDs and were also categorized into low, moderate and high groups. Analysis of variance was used to compare the mean of the variables across the tertiles of $\mathrm{VO}_{2}$ max and muscle strength. Associations of body composition, maximal strength tests and maximal aerobic capacity were examined using Pearson correlation coefficients. Linear regression with parameter estimates was used to investigate the independent associations between $\mathrm{VO}_{2}$ max and muscle strength, as independent variables, and body composition and anthropometric measurements, as dependent variables, which were adjusted according to age, sex, physical activity score and smoking in three different models. Model 1 was adjusted based on age and sex, model 2 was adjusted for age, sex and physical activity score, and model 3 was adjusted for age, sex, physical activity score and smoking. A $p$-value less than 0.05 was considered significant.

\section{Ethical consideration}

This study was approved by the local ethics review boards at the Tehran University of Medical Sciences (Ethic Number: R.TUMS.VCR.REC.1396.4306). All participants signed a written informed consent prior to the start of the study.

\section{Results}

The status of anthropometric measurements and physical fitness of the participants based on their level of CRF and muscle strength is shown in Table 1 . Significant associations were found between levels of $\mathrm{VO}_{2}$ max and height $(p<0.001)$, BMI $(p=0.004)$, FFM $(p<0.001)$, skeletal muscle mass (SMM) $(p<0.001)$, LBM $(p<0.001), \operatorname{FM}(p<0.001), \operatorname{BF} \%(p<0.001)$, visceral fat area (VFA) $(p<0.001)$, waist hip ratio (WHR) $(p<0.001)$, basal metabolic rate (BMR) $(p<0.001)$, abdominal fat $(p<0.001)$, obesity degree $(p=0.001)$, WC $(p=0.001)$, respiratory exchange ratio (RER) $(p=0.001)$ and heart rate $(p=0.004)$. Moreover, significant associations were found between levels of muscular fitness with height $(p<0.001)$, weight $(p<0.001), \operatorname{FFM}(p<0.001), \operatorname{SMM}(p<0.001), \operatorname{LBM}(p<0.001), \mathrm{WC}$ $(p<0.001), \operatorname{BMR}(p<0.001), \mathrm{BF} \%(p<0.001)$, VFA $(p=0.001)$, $\operatorname{BMI}(p=0.008), \operatorname{FM}(p=0.006)$, obesity degree $(p=0.029)$, WHR $(p=0.004)$ and $\operatorname{RER}(p=0.039)$.

The correlation between demographic and anthropometric measurements with $\mathrm{VO}_{2}$ max and muscle strength of the participants is presented in Table 2. Age was negatively correlated with $\mathrm{VO}_{2} \max (r=-0.393, p=0.001)$, but it was not correlated with muscle strength $(r=-0.035, p=0.562)$. In addition, a significant correlation was found between $\mathrm{VO}_{2}$ max with FFM $(r=0.369, p<0.001)$, LBM $(r=0.402, p<0.001)$ and SMM $(r=0.390$, $p<0.001)$. In addition, muscle strength had a significant correlation with FM $(r=-0.155, p=0.011)$, BMI $(r=-0.155, p=0.011)$, VFA $(r=-0.232, p=0.001)$, WC $(r=-0.311, p=0.001)$, WHR $(r=-0.204, p=0.011)$, RER $(r=0.171, p=0.001), \mathrm{BM}(r=0.548$, $p=0.001), \mathrm{BF} \%(r=-0.563, p=0.001), \mathrm{BMR}(r=0.676, p=0.001)$, height $(r=0.747, p=0.001)$, FFM $(r=0.821, p=0.001)$, SMM $(r=0.841, p=0.001)$ and LBM $(r=0.834, p=0.001)$.

The association between anthropometric measurements with $\mathrm{VO}_{2}$ max and muscular fitness after adjustments of age, sex, physical activity, and smoking are presented in Table 3. The association of $\mathrm{VO}_{2} \max \left(\mathrm{ml} \times \mathrm{min}^{-1} \times \mathrm{kg}^{-1}\right)$ with FFM $(p=0.164)$, $\operatorname{SMM}(p=0.145), \operatorname{LBM}(p=0.691)$, and $\operatorname{BMR}(p=0.262)$ was disappeared after adjustments. However, the association of $\mathrm{VO}_{2}$ max with other variables including FFM $(p<0.001)$, SMM $(p<0.001)$, LBM $(p<0.001)$, and BMR $(p<0.001)$ remained significant after adjustment for all confounders. In the case of muscle strength, adjustments disappeared its associations with FM ( $p=0.362)$ and VFA $(p=0.794)$. Whereas, the association of muscle strength with BMI, WC, WHR, RER, BM, BF\%, BMR, height, FFM, SMM, and LBM remained significant. 


\begin{tabular}{|c|c|c|c|c|c|c|c|c|c|}
\hline & \multicolumn{4}{|c|}{$\mathrm{VO}_{2}$ max, $\mathrm{mL} \cdot \mathrm{kg}^{-1} \cdot \mathrm{min}^{-1}$} & \multirow[b]{2}{*}{$p$} & \multicolumn{3}{|c|}{ Muscular strength, $\mathrm{kg}$} & \multirow[b]{2}{*}{$p$} \\
\hline & \multicolumn{2}{|c|}{ Low $(n=94)$} & $\begin{array}{l}\text { Moderate } \\
(n=123)\end{array}$ & High $(n=53)$ & & Low $(n=90)$ & $\begin{array}{l}\text { Moderate } \\
(n=90)\end{array}$ & High $(n=90)$ & \\
\hline Age (year) & \multicolumn{2}{|c|}{$35 \pm 12$} & $38 \pm 13$ & $36 \pm 14$ & 0.265 & $38 \pm 15$ & $36 \pm 13$ & $37 \pm 12$ & 0.541 \\
\hline Height $(\mathrm{cm})$ & \multicolumn{2}{|c|}{$165.84 \pm 9.94$} & $167.78 \pm 9.24$ & $173.15 \pm 10.04$ & $<0.001$ & $160.61 \pm 6.6$ & $166.94 \pm 7.52$ & $176.84 \pm 8.02$ & $<0.001$ \\
\hline Weight (kg) & \multicolumn{2}{|c|}{$73.7 \pm 19.66$} & $72.51 \pm 14.81$ & $71.68 \pm 11.26$ & 0.745 & $66.25 \pm 14.81$ & $68.64 \pm 12.17$ & $83.73 \pm 15.31$ & $<0.001$ \\
\hline BMI $\left(\mathrm{kg} / \mathrm{m}^{2}\right)$ & \multicolumn{2}{|c|}{$26.58 \pm 5.8$} & $25.58 \pm 4.05$ & $23.95 \pm 3.13$ & 0.004 & $25.69 \pm 5.65$ & $24.56 \pm 3.86$ & $26.74 \pm 4.2$ & 0.008 \\
\hline FFM (kg) & \multicolumn{2}{|c|}{$47.05 \pm 12.44$} & $49.83 \pm 12.44$ & $56.37 \pm 11.44$ & $<0.001$ & $41.12 \pm 6.85$ & $46.7 \pm 9.06$ & $62.77 \pm 9.92$ & $<0.001$ \\
\hline SMM & \multicolumn{2}{|c|}{$25.81 \pm 7.41$} & $27.81 \pm 7.14$ & $31.61 \pm 6.95$ & $<0.001$ & $22.27 \pm 4.11$ & $25.96 \pm 4.75$ & $35.45 \pm 5.9$ & $<0.001$ \\
\hline LBM & \multicolumn{2}{|c|}{$43.83 \pm 11.04$} & $47.42 \pm 11.18$ & $53.17 \pm 10.77$ & $<0.001$ & $38.54 \pm 6.22$ & $44.45 \pm 7.46$ & $59.05 \pm 8.88$ & $<0.001$ \\
\hline $\mathrm{FM}(\mathrm{kg})$ & \multicolumn{2}{|c|}{$26.64 \pm 10.93$} & $22.33 \pm 7.32$ & $15.47 \pm 6.02$ & $<0.001$ & $25.11 \pm 10.22$ & $21.47 \pm 7.66$ & $21.06 \pm 9.83$ & 0.006 \\
\hline PBF (\%) & \multicolumn{2}{|c|}{$35.3 \pm 8.25$} & $30.72 \pm 7.76$ & $21.75 \pm 8.28$ & $<0.001$ & $36.74 \pm 7.71$ & $30.57 \pm 7.73$ & $24.43 \pm 8.31$ & $<0.001$ \\
\hline VFA $\left(\mathrm{cm}^{2}\right)$ & \multicolumn{2}{|c|}{$127.57 \pm 53.86$} & $102.32 \pm 38.81$ & $67.62 \pm 32.39$ & $<0.001$ & $123.23 \pm 51.7$ & $97.89 \pm 42.01$ & $92.6 \pm 46.65$ & $<0.001$ \\
\hline Obesity degree & \multicolumn{2}{|c|}{$123.22 \pm 26.49$} & $117.39 \pm 18.12$ & $109.43 \pm 13.96$ & 0.001 & $119.51 \pm 26.13$ & $113.3 \pm 17.61$ & $121.4 \pm 18.91$ & 0.029 \\
\hline $\begin{array}{l}\text { Abdominal fat } \\
(\mathrm{kg})\end{array}$ & \multicolumn{2}{|c|}{$13.17 \pm 5.17$} & $11.4 \pm 3.84$ & $7.89 \pm 3.35$ & $<0.001$ & $12.2 \pm 4.83$ & $10.78 \pm 4.01$ & $11.1 \pm 5.05$ & 0.103 \\
\hline WC $(\mathrm{cm})$ & \multicolumn{2}{|c|}{$92.02 \pm 15.07$} & $90.14 \pm 11.3$ & $84.26 \pm 8.53$ & 0.001 & $87.22 \pm 13$ & $87.1 \pm 10.6$ & $94.86 \pm 12.67$ & $<0.001$ \\
\hline WHR & \multicolumn{2}{|c|}{$0.92 \pm 0.07$} & $0.91 \pm 0.06$ & $0.86 \pm 0.06$ & $<0.001$ & $0.89 \pm 0.06$ & $0.9 \pm 0.06$ & $0.92 \pm 0.07$ & 0.004 \\
\hline BMR & \multicolumn{2}{|c|}{$1395.17 \pm 274.39$} & $1424.42 \pm 308.09$ & $1587.62 \pm 246.99$ & $<0.001$ & $1258.76 \pm 147.78$ & $1397.92 \pm 179.69$ & $1685.44 \pm 330.86$ & $<0.001$ \\
\hline Heart rate & \multicolumn{2}{|c|}{$166.89 \pm 22.58$} & $171.67 \pm 18.86$ & $178.5 \pm 15.36$ & 0.004 & $168.36 \pm 21.31$ & $172.49 \pm 18.38$ & $173.37 \pm 20.47$ & 0.235 \\
\hline RER & \multicolumn{2}{|c|}{$1.07 \pm 0.05$} & $1.09 \pm 0.05$ & $1.11 \pm 0.05$ & 0.001 & $1.08 \pm 0.05$ & $1.09 \pm 0.05$ & $1.1 \pm 0.05$ & 0.039 \\
\hline \multirow[t]{2}{*}{ Sex } & $M$ & \begin{tabular}{|l|}
21 \\
$(17.9 \%)$ \\
\end{tabular} & 57 (48.7\%) & 39 (33.3\%) & \multirow{2}{*}{$<0.001$} & $4(3.4 \%)$ & $28(23.5 \%)$ & 87 (73.1\%) & \multirow{2}{*}{$<0.001$} \\
\hline & $F$ & \begin{tabular}{|l|}
73 \\
$(47.7 \%)$ \\
\end{tabular} & 66 (43.1\%) & $14(9.2 \%)$ & & $86(57 \%)$ & 62 (41.1\%) & $3(2 \%)$ & \\
\hline \multirow[t]{3}{*}{ Activity score } & L & $43(43 \%)$ & 47 (47\%) & $10(10 \%)$ & \multirow{3}{*}{$<0.001$} & 38 (38.8\%) & $33(33.7 \%)$ & 27 (27.6\%) & \multirow{3}{*}{0.009} \\
\hline & $M$ & \begin{tabular}{|l|}
39 \\
$(35.1 \%)$ \\
\end{tabular} & 51 (45.9\%) & 21 (18.9\%) & & $38(33.6 \%)$ & $41(36.3 \%)$ & 34 (30.1\%) & \\
\hline & v & \begin{tabular}{|l|}
10 \\
$(18.2 \%)$
\end{tabular} & 23 (41.8\%) & $22(40 \%)$ & & $10(18.2 \%)$ & $16(29.1 \%)$ & $29(52.7 \%)$ & \\
\hline
\end{tabular}

BMI - body mass index, FFM - fat free mass, SMM - skeletal muscle mass, LBM - lean body mass, PBF - percent body fat, VFA - visceral far area, WC - waist circumference, WHR - waist hip ratio, BMR - basal metabolic rate, RER - respiratory exchange ratio, $\mathrm{M}$ - male, $\mathrm{F}$ - female, $\mathrm{Y}$ - yes, $\mathrm{N}$ - no, $\mathrm{L}$ - low, $\mathrm{M}$ - moderate, $\mathrm{V}$ - vigorous.

\begin{tabular}{|c|c|c|c|c|}
\hline \multirow[t]{3}{*}{ Variables } & \multicolumn{4}{|c|}{ Physical fitness tests } \\
\hline & \multicolumn{2}{|c|}{$\mathrm{VO}_{2} \max , \mathrm{mL} \cdot \mathrm{kg}^{-1} \cdot \min ^{-1}$} & \multicolumn{2}{|c|}{ Muscular fitness, kg } \\
\hline & $r$ & $p$ & $r$ & $p$ \\
\hline Age (year) & -0.393 & 0.001 & -0.035 & 0.562 \\
\hline Height $(\mathrm{cm})$ & 0.500 & 0.001 & 0.747 & 0.001 \\
\hline Weight (kg) & -0.68 & 0.267 & 0.548 & 0.001 \\
\hline BMI $\left(\mathrm{kg} / \mathrm{m}^{2}\right)$ & -0.405 & 0.001 & 0.155 & 0.011 \\
\hline FFM (kg) & 0.369 & 0.001 & 0.821 & 0.001 \\
\hline SMM & 0.390 & 0.001 & 0.841 & 0.001 \\
\hline LBM & 0.402 & 0.001 & 0.834 & 0.001 \\
\hline FM (kg) & -0.608 & 0.001 & -0.155 & 0.011 \\
\hline PBF (\%) & -0.748 & 0.001 & -0.563 & 0.001 \\
\hline VFA $\left(\mathrm{cm}^{2}\right)$ & -0.653 & 0.001 & -0.232 & 0.001 \\
\hline Obesity degree & 0.449 & 0.001 & 0.101 & 0.098 \\
\hline Abdominal fat $(\mathrm{kg})$ & -0.581 & 0.001 & -0.066 & 0.277 \\
\hline$W C(\mathrm{~cm})$ & -0.318 & 0.001 & 0.311 & 0.001 \\
\hline WHR & -0.381 & 0.001 & 0.204 & 0.001 \\
\hline BMR & 0.309 & 0.001 & 0.676 & 0.001 \\
\hline Heart Rate & 0.417 & 0.001 & 0.119 & 0.064 \\
\hline RER & 0.312 & 0.001 & 0.171 & 0.005 \\
\hline
\end{tabular}

BMI - body mass index, FFM - fat free mass, SMM - skeletal muscle mass, LBM - lean body mass, PBF - percent body fat, VFA - visceral far area, WC - waist circumference, WHR - waist hip ratio, BMR - basal metabolic rate, RER - respiratory exchange ratio. 


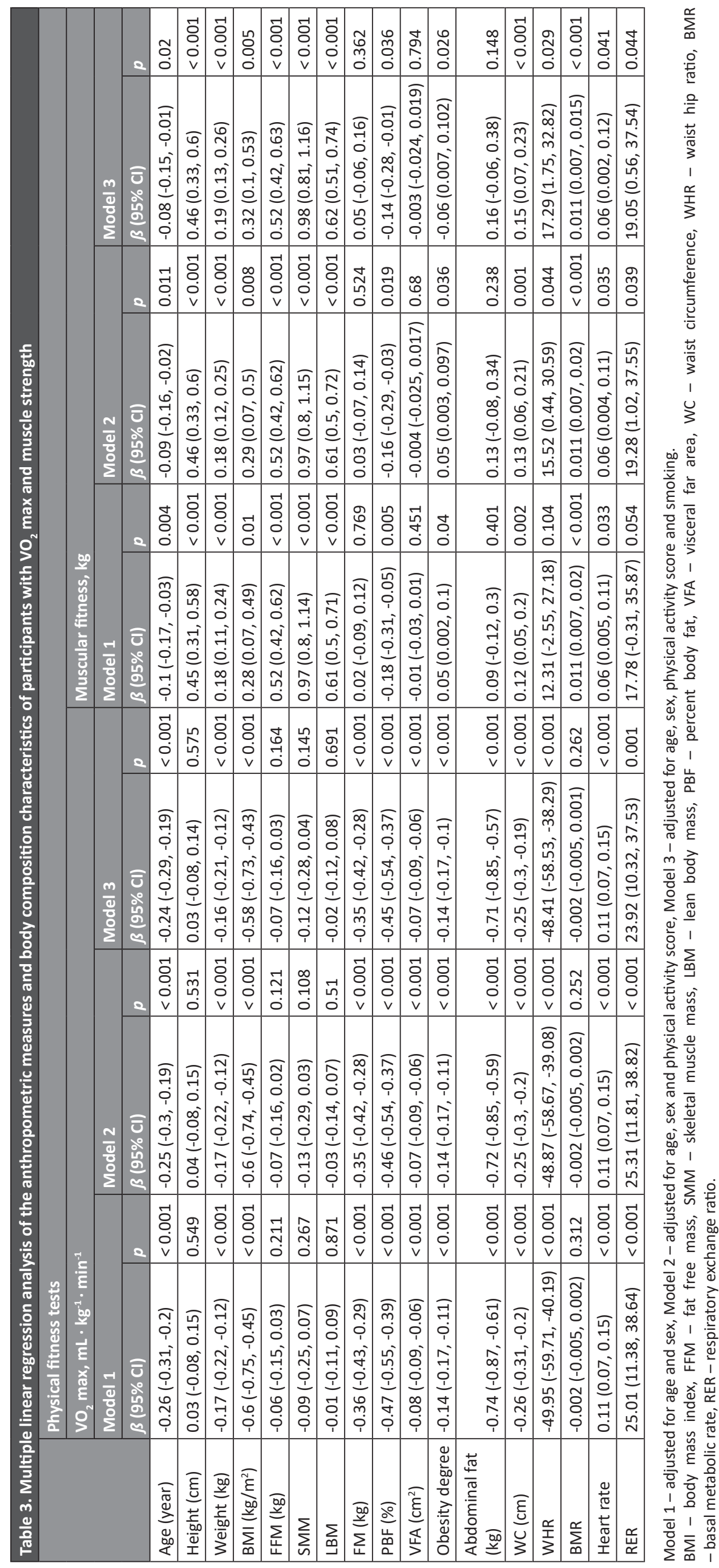




\section{Discussion}

The results of this study revealed that CRF was negatively associated with BMI, FM, VFA, abdominal fat, obesity degree, WC, WHR and age before and after adjustment and was positively associated with height, FFM, BMR, RER and maximal heart rate. There was a significant association between absolute $\mathrm{VO}_{2}$ $\max \left(I \times \min ^{-1}\right)$ and FFM, SMM, LBM and BMR, even after adjustment for all confounders. Similar to these results in adults, several studies have reported that overweight children are categorized at a poor $\mathrm{VO}_{2}$ max level compared to healthy weight children [40,41]. The association observed between maximal oxygen uptake and body composition measurements was in line with a recently published report by Goran et al. in older adults [42]. They revealed that participants with higher body FM appear to have a lower aerobic capacity. Rump et al. [43], in a birth-cohort among young prepubescent children, identified that $\mathrm{FM}$ was negatively associated with $\mathrm{VO}_{2}$ max and was positively associated with submaximal heart rate. Fat-free mass was positively related to absolute oxygen uptake. Moreover, they observed a significant association between the relative value of CRF and weight, BMI and WC [44]. Brand et al. reported a significant association between CRF and overweight and obesity in boys, while this association was marginally insignificant in girls. An increase in body fat percentage, especially visceral fat, may impair the proper functioning of the cardiovascular system, regardless of the status of BMI. The higher prevalence of "apple" shaped obesity in men (i.e. central obesity) may explain the stronger relationship we observed between $\mathrm{FM}$ and $\mathrm{VO}_{2}$ max in men. Moreover, the effects of BF on CRF can be mediated by mean arterial pressure (MAP). A higher percentage of $B F$ is associated with higher MAP [45]. However, some other factors, such as genetics and gender, may have an influence on the status of body composition and CRF [31, 46-49].

Moreira et al. [31] indicated that BMR and VFA significantly affect the variety of CRF, regardless of menopause and age. The presence of CRF levels $>30.94 \mathrm{ml} / \mathrm{kg} / \mathrm{min}$ was related to low FM and ameliorated muscle status, and postmenopausal women with $\mathrm{VO}_{2} \max <26.87 \mathrm{ml} / \mathrm{kg} / \mathrm{min}$ had higher VFA. Mota et al. [50] found a significant difference in the CRF of an obese group of females, while the association was not significant in males. Kim et al. [51], in a longitudinal study, reported that CRF was significantly related with the risk of overweight in girls but not in boys. This may be due to a higher BMI and muscle mass in boys than in girls.

In the present study, maximal strength was also positively associated with height, weight, BMI, FFM, SMM, LBM, WC, WHR and BMR. There are positive significant relationships among these parameters, even after adjusting for confounding factors. Nevertheless, we found a negative association between muscular fitness and $\mathrm{FM}, \mathrm{BF} \%$ and VFA, which disappeared after adjustments. Vaara et al. [29] concluded that muscle strength index had low correlations with BM, WC, BMI and FM. However, FFM had a positive moderate association with maximal strength test scores. In contrast, Woo et al., in a cross sectional study on 4,000 men and women $\geq 65$ years of age, indicated that fat mass and $\mathrm{BMI}$ are main factors contributing to physical performance [52].

Another study [53] indicated that LBM was positively associated with muscular strength. A $1 \mathrm{~kg}$ increase in birth weight corresponded in men to a $4.1 \mathrm{~kg}(95 \% \mathrm{Cl}: 3.1,5.1)$ and in women to a $2.9 \mathrm{~kg}(2.1,3.6)$ increase in adult lean mass. BF\% was negatively associated with muscle strength in men. Ageing and smoking were other factors which related to poor muscular fitness.

In line with our findings, Payette et al. [54] revealed that grip strength was significantly correlated with FFM in frail elderly women, particularly in those without any pain. Likewise, Estrada et al. [55] reported that muscular handgrip strength was related to lower-limb, upper-limb and total SMM, even considering the impact of body mass or height.

In the present study, men showed better and longer aerobic power and gained greater maximal oxygen consumption along with higher muscle strength than women. The differences could be explained by sex-related discrepancies in body composition, FM and FFM [56, 57].

Several studies have indicated that the higher muscular strength in men may result from the fact that women have lower lean tissue mass. Furthermore, the higher muscle strength in men may be caused by larger muscular fibers, which derive from a biological difference rather than a difference in activity score [58].

However, the present study had some limitations. First, this study was a cross-sectional study with a relatively small sample size. More accurate CRF tests could be used, but they require expensive equipment, take too much time and may cause some risks for older adults [59].

\section{Conclusions}

Relative $\mathrm{VO}_{2}$ max was related to $\mathrm{FM}$ and $\mathrm{BF} \%$. Muscular fitness was associated with FM. It can be concluded that the body composition analysis may be essential to predict CRF and muscle strength test results. High FM was associated with poor CRF, and low fat-free mass was related to poor muscle strength. The differences between the $\mathrm{VO}_{2}$ max and muscle strength of men and women can be explained by differences in body composition, especially in FFM.

Acknowledgements. This study was conducted at the school of Nutrition and Food Sciences and the Tehran University of Medical Sciences, Tehran, Iran. We would like to acknowledge the staff of the mentioned centers for their kind cooperation.

Source of funding: This study was funded by the Tehran University of Medical Sciences.

Conflicts of interest: The authors declare no conflicts of interest.

\section{Refrences}

1. Bevier WC, Wiswell RA, Pyka G, et al. Relationship of body composition, muscle strength, and aerobic capacity to bone mineral density in older men and women. J Bone Miner Res 1989; 4(3): 421-432.

2. Haghshenas R, Jamshidi Z, Doaei $S$, et al. The effect of a high-intensity interval training on plasma vitamin $D$ level in obese male adolescents. Indian J Endocrinol Metab 2019; 23(1): 72-75.

3. Williams MA, Haskell WL, Ades PA, et al. Resistance exercise in individuals with and without cardiovascular disease: 2007 update: a scientific statement from the American Heart Association Council on Clinical Cardiology and Council on Nutrition, Physical Activity, and Metabolism. Circulation 2007; 116(5): 572-584.

4. Fujita Y, Nakamura Y, Hiraoka J, et al. Physical-strength tests and mortality among visitors to health-promotion centers in Japan. $J$ Clin Epidemiol 1995; 48(11): 1349-1359.

5. Kodama S, Saito K, Tanaka S, et al. Cardiorespiratory fitness as a quantitative predictor of all-cause mortality and cardiovascular events in healthy men and women: a meta-analysis. JAMA 2009; 301(19): 2024-2035. 
6. Metter EJ, Talbot LA, Schrager M, et al. Skeletal muscle strength as a predictor of all-cause mortality in healthy men. $J$ Gerontol A Biol Sci Med Sci 2002; 57(10): B359-B365.

7. Phillips P. Grip strength, mental performance and nutritional status as indicators of mortality risk among female geriatric patients. Age Ageing 1986; 15(1): 53-56.

8. Wei M, Kampert JB, Barlow CE, et al. Relationship between low cardiorespiratory fitness and mortality in normal-weight, overweight, and obese men. JAMA 1999; 282(16): 1547-1553.

9. Blair SN, Kampert JB, Kohl HW, et al. Influences of cardiorespiratory fitness and other precursors on cardiovascular disease and allcause mortality in men and women. JAMA 1996; 276(3): 205-210.

10. Chatterjee S, Chatterjee P. Prediction of maximal, oxygen consumption from body mass, height and body surface area. Indian J Physiol Pharmacol 2006; 50(2): 181-186.

11. Kumar S. Muscle strength. Boca Raton: CRC Press Boca Raton; 2004.

12. Zwart D, Arjan H, Dekker J, et al. Factors associated with upper leg muscle strength in knee osteoarthritis: a scoping review. $J$ Rehabil Med 2018; 50(2): 140-150.

13. Norman K, Stobäus N, Gonzalez MC, et al. Hand grip strength: outcome predictor and marker of nutritional status. Clin Nutr 2011; 30(2): 135-142.

14. Bouchard CE, Shephard RJ, Stephens TE, eds. Physical activity, fitness, and health: international proceedings and consensus statement. International Consensus Symposium on Physical Activity, Fitness, and Health, 2nd, May, 1992, Toronto, ON, Canada. Toronto: Human Kinetics Publishers; 1994.

15. Kalantari N, Mohammadi NK, Rafieifar S, et al. Indicator for success of obesity reduction programs in adolescents: body composition or body mass index? Evaluating a school-based health promotion project after 12 weeks of intervention. Int J Prev Med 2017; 8: 73, doi: 10.4103/ijpvm.IJPVM_306_16.

16. Doaei S, Malmir H, Kalantari N, et al. Body mass index (BMI) was associated with mental health, but it had no association with selfesteem in Iranian girls: a case-control study. Acta Medica Mediterranea 2018; 34(4): 1017-1025.

17. Esco MR, Olson MS, Williford HN. The relationship between selected body composition variables and muscular endurance in women. Res Q Exerc Sport 2010; 81(3): 272-277.

18. Fogelholm M, Malmberg J, Suni J, et al. Waist circumference and BMI are independently associated with the variation of cardio-respiratory and neuromuscular fitness in young adult men. Int J Obes 2006; 30(6): 962-969.

19. Doaei S, Gholamalizadeh M, Entezari MH. Maternal self-efficacy and feeding practices in children aged 3-6 years. Iran J Psychiatry 2015; 10(4): 278-284.

20. Lahouti M, Zavoshy R, Noroozi M, et al. Dietary patterns and depressive symptoms among Iranian women. J Health Psychol 2020, doi: $10.1177 / 1359105320909888$.

21. Kalantari N, Doaei S, Gordali M, et al. The association between dairy intake, simple sugars and body mass index with expression and extent of anger in female students. Iran J Psychiatry 2016; 11(1): 43-50.

22. Wong SL, Katzmarzyk PT, Nichaman MZ, et al. Cardiorespiratory fitness is associated with lower abdominal fat independent of body mass index. Med Sci Sport Exer 2004; 36(2): 286-291.

23. Tzankoff S, Norris A. Effect of muscle mass decrease on age-related BMR changes. J Appl Physiol Respir Environ Exerc Physiol 1977; 43(6): 1001-1006.

24. Duvigneaud N, Matton L, Wijndaele K, et al. Relationship of obesity with physical activity, aerobic fitness and muscle strength in Flemish adults. J Sports Med Phys Fitness 2008; 48(2): 201-210.

25. Mota J, Guerra S, Leandro C, et al. Association of maturation, sex, and body fat in cardiorespiratory fitness. Am J Hum Biol 2002; 14(6): 707-712.

26. Koster A, Ding J, Stenholm S, et al. Does the amount of fat mass predict age-related loss of lean mass, muscle strength, and muscle quality in older adults? J Gerontol A Biol Sci Med Sci 2011; 66A(8): 888-895.

27. Church TS, Kuk JL, Ross R, et al. Association of cardiorespiratory fitness, body mass index, and waist circumference to nonalcoholic fatty liver disease. Gastroenterology 2006; 130(7): 2023-2030.

28. Hardy R, Cooper R, Sayer AA, et al. Body mass index, muscle strength and physical performance in older adults from eight cohort studies: the HALCyon programme. PLoS ONE 2013; 8(2): e56483.

29. Vaara JP, Kyröläinen H, Niemi J, et al. Associations of maximal strength and muscular endurance test scores with cardiorespiratory fitness and body composition. J Strength Cond Res 2012; 26(8): 2078-2086.

30. Goodpaster BH, Park SW, Harris TB, et al. The loss of skeletal muscle strength, mass, and quality in older adults: the health, aging and body composition study. J Gerontol A Biol Sci Med Sci 2006; 61(10): 1059-1064.

31. Moreira H, Passos B, Rocha J, et al. Cardiorespiratory fitness and body composition in postmenopausal women. J Hum Kinet 2014; 43: 139-148.

32. Khanna G, Majumdar P, Saha M, et al. Cardiorespiratory fitness and body composition in Indian children of 10-16 years. In: Parizková J, Hills AP, eds. Physical fitness and nutrition during growth. Med Sport Sci 1998; 43: 132-144, doi: 10.1159/000061751.

33. Burns R, Hannon JC, Brusseau TA, et al. Indices of abdominal adiposity and cardiorespiratory fitness test performance in middle-school students. J Obes 2013; 2013, doi: 10.1155/2013/912460.

34. Bruce RA, Pearson R, Lovejoy FW, et al. Variability of respiratory and circulatory performance during standardized exercise. Int J Sports Med 1985; 6(4): 197-201.

35. Medbo JI, Mamen A, Welde B, et al. Examination of the metamax I and II oxygen analysers during exercise studies in the laboratory. Scand J Clin Lab Invest 2002; 62: 585-598.

36. Bohannon RW. Hand-grip dynamometry provides a valid indication of upper extremity strength impairment in home care patients. $J$ Hand Ther 1998; 11(4): 258-260.

37. Schiavo L, Scalera G, Pilone V, et al. Fat mass, fat-free mass, and resting metabolic rate in weight-stable sleeve gastrectomy patients compared with weight-stable nonoperated patients. Surg Obes Relat Dis 2017; 13(10): 1692-1699.

38. Craig CL, Marshall AL, Sjöström M, et al. International physical activity questionnaire: 12 -country reliability and validity. Med Sci Sports Exerc 2003; 35(8): 1381-1395.

39. Chatrath R, Shenoy R, Serratto M, et al. Physical fitness of urban American children. Pediatr Cardiol 2002; 23(6): 608-612.

40. Davarzani S, Babaei N, Ebaditabar M, et al. Associations of physical activity with cardiorespiratory fitness, muscle strength, and body composition. Pediatr Endocrinol Diabetes Metab 2020; 26(4): 183-191.

41. Ward D, Evans R. Physical activity, aerobic fitness and obesity in children. Med Exerc Nutr Health 1995; 4(3): 3-16.

42. Goran M, Fields D, Hunter G, et al. Total body fat does not influence maximal aerobic capacity. Int J Obes Relat Metab Disord 2000; 24(7): 841-848. 
43. Rump P, Verstappen F, Gerver W, et al. Body composition and cardiorespiratory fitness indicators in prepubescent boys and girls. Int J Sports Med 2002; 23(1): 50-54.

44. Bellefeuille P, Robillard M-E, Ringuet M-E, et al. Comparison between several muscle strength and cardiorespiratory fitness indices with body composition and energy expenditure in obese postmenopausal women. Int J Sports Med 2013; 34(3): 258-262.

45. Brand C, Dias AF, Fochesatto CF, et al. The role of body fat in the relationship of cardiorespiratory fitness with cardiovascular risk factors in Brazilian children. Motriz: Revista de Educação Física 2018; 24(4), doi: 10.1590/s1980-6574201800040015.

46. Kalantari N, Mohammadi NK, Izadi P, et al. A complete linkage disequilibrium in a haplotype of three SNPs in Fat Mass and Obesity associated (FTO) gene was strongly associated with anthropometric indices after controlling for calorie intake and physical activity. BMC Med Genet 2018; 19(1): 146, doi: 10.1186/s12881-018-0664-z.

47. Gholamalizadeh M, Doaei S, Akbari ME, et al. Influence of fat mass-and obesity-associated genotype, body mass index, and dietary intake on effects of iroquois-related homeobox 3 gene on body weight. Chin Med J 2018; 131(17): 2113-2122.

48. Doaei S, Kalantari N, Izadi P, et al. Interactions between macro-nutrients' intake, FTO and IRX3 gene expression, and FTO genotype in obese and overweight male adolescents. Adipocyte 2019; 8(1): 386-391.

49. Doaei S, Hajiesmaeil M, Aminifard A, et al. Effects of gene polymorphisms of metabolic enzymes on the association between red and processed meat consumption and the development of colon cancer; a literature review. J Nutr Sci 2018; 7: e26, doi: 10.1017/ jns.2018.17.

50. Mota J, Flores L, Flores L, et al. Relationship of single measures of cardiorespiratory fitness and obesity in young schoolchildren. $\mathrm{Am} \mathrm{J}$ Human Biol 2006; 18(3): 335-341.

51. Kim J, Must A, Fitzmaurice GM, et al. Relationship of physical fitness to prevalence and incidence of overweight among schoolchildren. Obes Res 2005; 13(7): 1246-1254.

52. Woo J, Leung J, Kwok T. BMI, body composition, and physical functioning in older adults. Obesity 2007; 15(7): $1886-1894$.

53. Ylihärsilä H, Kajantie E, Osmond C, et al. Birth size, adult body composition and muscle strength in later life. Int J Obesity 2007; 31(9): 1392-1399.

54. Payette $\mathrm{H}$, Hanusaik N, Boutier V, et al. Muscle strength and functional mobility in relation to lean body mass in free-living frail elderly women. Eur J Clin Nutr 1998; 52(1): 45-53.

55. Estrada M, Kleppinger A, Judge JO, et al. Functional impact of relative versus absolute sarcopenia in healthy older women. J Am Geriatr Soc 2007; 55(11): 1712-1719.

56. Boileau R, Lohman TG, Slaughter M, et al. Hydration of the fat-free body in children during maturation. Hum Biol 1984; 56(4): 651-666.

57. Fomon SJ, Haschke F, Ziegler EE, et al. Body composition of reference children from birth to age 10 years. Am J Clin Nutr 1982; 35(5 Suppl): 1169-1175.

58. Miller AEJ, MacDougall J, Tarnopolsky M, et al. Gender differences in strength and muscle fiber characteristics. Eur J Appl Physiol Occup Physiol 1993; 66(3): 254-262.

59. Blair SN, Kampert JB, Kohl HW, et al. Influences of cardiorespiratory fitness and other precursors on cardiovascular disease and allcause mortality in men and women. JAMA 1996; 276(3): 205-210.

Tables: 3

Figures: 0

References: 59

Received: 21.05.2020

Reviewed: 26.05 .2020

Accepted: 1.09 .2020

Address for correspondence:

Sakineh Shab-Bidar, PhD, Assoc. Prof.

Department of Community Nutrition

School of Nutritional Sciences and Dietetics

Tehran University of Medical Sciences (TUMS)

No 44, Hojjat-dost Alley, Naderi St., Keshavarz Blvd

Tehran

Iran

Tel.: +989111376516

E-mail: s_shabbidar@tums.ac.ir 